\title{
Risk Factors for pT3a Prostate Cancer in the Patients With cT1-2 on Preoperative Multiparametric Magnetic Resonance Imaging
}

\author{
Kyung Hwan Kim', Ja Yoon $\mathrm{Ku}^{1}$, Won Young Park², Seung Baek Hong ${ }^{3}$, \\ Suk $\mathrm{Kim}^{3}$, Hong $\mathrm{KoO} \mathrm{Ha}^{1,4}$
}

\author{
${ }^{1}$ Department of Urology, Pusan National University Hospital, Pusan National University School of Medicine, Busan, Korea \\ ${ }^{2}$ Department of Pathology, Pusan National University Hospital, Pusan National University School of Medicine, Busan, Korea \\ ${ }^{3}$ Department of Radiology, Pusan National University Hospital, Pusan National University School of Medicine, Busan, Korea \\ ${ }^{4}$ Biomedical Research Institute, Pusan National University Hospital, Busan, Korea
}

\begin{abstract}
Purpose: Our study aimed to evaluate the predictive value of multiparametric magnetic resonance imaging (mpMRI) to assess the capsular extension of prostate cancer. Risk factors for extraprostatic invasion or microscopic invasion of bladder neck (PT3a stage) in the cases showing localized disease findings on MRI scan were also identified. Materials and Methods: We identified 198 patients who underwent robot-assisted or conventional laparoscopic radical prostatectomy in our institute between June, 2016 and May, 2018. Altogether, 170 patients with preoperational mpMRI scans and complete pathologic data were included. Positive and negative predictive values of mpMRI to detect capsular extension were estimated. Chi-square test was performed for ratio variables. Logistic regression analysis was performed to identify capsular invasion risk factors.

Results: Median age and prostate-specific antigen level were 68 years and $7.5 \mathrm{ng} / \mathrm{mL}$, respectively. Based on findings, 16 patients $(9.4 \%)$ were identified as CT3a stage (unilateral or bilateral extraprostatic extension), whereas 37 patients $(21.8 \%)$ as pT3a stage. Positive and negative predictive values of mpMRI to detect capsular extension were $75.0 \%$ and $73.9 \%$, respectively. In the logistic regression analysis, positive biopsy core rate was the significant predictor for pT3a disease in the patients with negative capsular invasion findings on mpMRI ( $p<0.001)$. According to receiver-operating characteristic curve (area under the curve $=0.691, p=0.001$ ), the positive biopsy core rate of 0.275 was the best threshold.

Conclusions: Multiparametric MRI is an appropriate test to predict pT3a disease preoperatively. The patients with positive core rate over 0.275 may have pT3a diseases despite negative image findings. (Korean J Urol Oncol 2020;18:109-115)
\end{abstract}

Key Words: Biopsy $\cdot$ Magnetic resonance imaging $\cdot$ Prostatectomy $\cdot$ Tumor grading $\cdot$ Ultrasonography

Received February 25, 2020, Revised April 26, 2020,

Accepted May 11, 2020

Comesponding Author: Hong Koo Ha

Department of Urology, Pusan National University Hospital, Pusan National University School of Medicine, 179 Gudeok-Ro,

Seo-Gu, Busan 49241, Korea

E-mail: hongkooha@naver.com

Tel: +82-51-240-7351, Fax: +82-51-247-5443

ORCID: https://orcid.org/0000-0002-8240-7765

\section{INTRODUCTION}

Prostate cancer is one of the most frequently diagnosed malignancies worldwide. The International Agency for Research on Cancer estimated that 1,276,106 new cases were diagnosed globally in 2018. ${ }^{1}$ Early diagnosis and curative treatment may reduce the cancer mortality in the patients with intermediate and high risks. In the cases of low-risk disease, overdetection and overtreatment remain as

This is an Open Access article distributed under the terms of the Creative Commons Attribution Non-Commercial License (http://creativecommons.org/licenses/by-nc/4.0/) which permits unrestricted non-commercial use, distribution, and reproduction in any medium, provided the original work is properly cited. 2020 (C) Copyright The Korean Urological Oncology Society and The Korean Prostate Society. All Rights Reserved. 
significant concerns. The survival benefits of radical prostatectomy over watchful waiting were not identified in the patients with low risk disease. ${ }^{2,3}$ Moreover, active surveillance (AS) is a reasonable management option for the patients with low-risk prostate cancer. ${ }^{4}$

Magnetic resonance imaging (MRI) is commonly used in detecting and monitoring prostate cancer. The performance of multiparametric MRI (mpMRI) in evaluating prostate cancer has been demonstrated in several studies. ${ }^{5-7}$ The value of MRI scans with or without targeted biopsy in selecting eligible patients for AS and monitoring cancer progression in AS setting have been studied recently. ${ }^{8-11}$ However, researches on capsular invasion prediction of MRI scan are limited. Extracapsular extension (ECE) of prostate cancer, indicating the T3a stage, can be an important clinical factor in deciding therapeutic options for prostate cancer.

Thus, we aimed to investigate the value of mpMRI to predict the ECE of prostate cancer and identify the risk factors for pathological T3a stage in the cases of clinical T1-2 disease based on MRI findings.

\section{MATERIALS AND METHODS}

\section{Study Population}

Between June, 2016 and May, 2018, 198 consecutive patients who underwent robot-assisted or conventional laparoscopic radical prostatectomy by a single surgeon in our institute were identified. There were no differences in surgical techniques between robot-assisted or conventional laparoscopic radical prostatectomy groups. Excluding 28 men ineligible for this study, 170 patients with preoperational mpMRI scans and complete pathologic data were enrolled. Exclusion criteria were absence of preoperative MRI scan or prostate biopsy records, incompleteness of MRI findings, pathologic diagnosis other than prostatic adenocarcinoma, and history of surgical intervention or androgen deprivation therapy before radical prostatectomy.

\section{MRI Data Acquisition}

One hundred seventy patients underwent $3 \mathrm{~T}$ mpMRI scans preoperatively (Achieva TX, Philips Healthcare, Best, the Netherlands). Multiparametric MRI sequences included triplanar T2-weighted imaging and high b-value dif- fusion-weighted imaging with apparent diffusion coefficient maps. We did not conduct the endorectal coil scans. MRI scans were reviewed by 2 radiologists (SK and SBH) with 25 and 10 years of experiences, respectively. The prostatic lesions were evaluated based on the Prostate ImagingReporting and Data System (PI-RADS) version 2 guidelines. ${ }^{12-14}$ Any disagreement was resolved to consensus by interobserver discussion. ${ }^{15}$ Researchers encouraged the patients to evacuate the rectum before the MRI tests. To acquire accurate estimates of prostatic volume, the ellipsoidal formula was used. ${ }^{16}$

\section{Pathological Data Assessment}

The prostatic specimens, samples from preoperative prostate biopsy, and radical prostatectomy, were evaluated at the department of pathology in our institution. A single genitourinary pathologist (WYP) with 12 years of experience examined all the prostatic samples. Pathological stage was determined by cancer staging system (7th edition) of American Joint Committee on Cancer. ${ }^{17}$

\section{Prostate Biopsy Procedure}

Prostate biopsies were conducted by the urologists in the department of urology. Out of 170 patients, 152 patients underwent the transrectal ultrasound (TRUS)-guided systemic biopsy with 12 cores; 12>cores and 13 cores biopsies were performed in 14 and 4 patients, respectively. Patients underwent prostate biopsies under local anesthetic injection of 2 syringes of lubricant mixed with lidocaine into the rectum. Rectal enema and povidone-iodine solution preparation were administered to prevent any post-procedural infection. Affiniti 70 ultrasound system (Philips Healthcare) was used.

\section{Statistical Analysis}

Continuous variables were presented as median with interquartile range or mean with $95 \%$ confidence interval. Categorical parameters were listed as numbers with percentages. Chi-squared test was used for the analysis of the proportional data. For the risk factor analysis, logistic regression test was conducted. A receiver-operating characteristic (ROC) curve was plotted to calculate the cutoff value of the identified risk factor. Statistical significance was considered at $\mathrm{p}<0.05$. 


\section{Ethics Statement and Description of Participants}

This is an observational study conducted in a retrospective manner at a single tertiary referral hospital. The researchers abided by the Good Clinical Practices and the ethical principles of the Declaration of Helsinki. Patients' data were acquired from electronic medical records and picture archiving and communication systems. The Institutional Review Board of Pusan National University Hospital approved the protocol of this study (2002-006-087).

\section{RESULTS}

\section{Clinical Characteristics}

Clinical characteristics of 170 patients are listed in Table

Table 1. Clinical characteristics of study population $(n=170)$

\begin{tabular}{lcl}
\hline \multicolumn{1}{c}{ Characteristic } & \multicolumn{1}{c}{ Value } \\
\hline Age (yr), median (IQR) & $68(64-72)$ \\
PSA (ng/mL), median (IQR) & $7.5\left(5.6^{-12.2)}\right.$ \\
Prostate volume (mL), median (IQR) & $36.3(27.9-44.8)$ \\
PSAD (ng/mL $\left.{ }^{2}\right)$, median (IQR) & $0.23(0.15-0.38)$ \\
Prostate Biopsy & \\
$\quad$ Biopsy core number, mean (95\% CI) & $12.4(12.1-12.8)$ \\
Positive core number, mean $(95 \% \mathrm{CI})$ & $4.6(4.1-5.2)$ \\
Positive rate, mean (95\% CI) & $0.38(0.34-0.42)$ \\
Surgery & $93(54.7)$ \\
$\quad$ RARP (\%) & $77(45.3)$ \\
LRP (\%)
\end{tabular}

IQR: interquartile range, PSA: prostate-specific antigen, PSAD: PSA density, CI: confidence interval, RARP: robot-assisted laparoscopic radical prostatectomy, LRP: laparoscopic radical prostatectomy.
1. Median age, prostate-specific antigen (PSA) level, and PSA density (PSAD) are 68 years, $7.5 \mathrm{ng} / \mathrm{mL}$, and 0.23 $\mathrm{ng} / \mathrm{mL}^{2}$, respectively. The mean number of total biopsy and positive biopsy cores are 12.4 and 4.6 leading to the positive rate of 0.38 , respectively. Out of 170 patients, 93 $(54.7 \%)$ and 77 (45.3\%) underwent robot-assisted laparoscopic radical prostatectomy (RARP) and conventional laparoscopic radical prostatectomy (LRP), respectively. We cannot identify the significant differences of clinical characteristics between RARP and LRP groups. The ratios of confirmative R1 classification are similar between 2 groups (Table 2).

\section{Comparison of Clinical and Pathological T Stage}

The distributions of clinical and pathological $\mathrm{T}$ stage are demonstrated in Fig. 1. Based on the mpMRI findings, 136 $(80 \%)$ and 16 patients $(9.4 \%)$ are classified as clinical T1-2 and T3a stages, respectively. $104(61.2 \%)$ and 37 patients (21.8\%) belongs to pathological $\mathrm{T} 2$ and $\mathrm{T} 3 \mathrm{a}$ stage, respectively. Twenty-one patients are recategorized to $\mathrm{T} 3 \mathrm{a}$ stage after prostatectomy. The proportion of $\mathrm{T} 3 \mathrm{~b}$ stage is also higher in the pathological (28 patients, 16.5\%) than in the clinical stage distribution (18 patients, 10.6\%).

\section{International Society of Urological Pathology Grade of Prostate Specimen}

The International Society of Urological Pathology (ISUP) grade distribution of prostate biopsy samples and prostatectomy specimen is listed in Table 3. The ISUP grade distributions between 2 type of prostate specimen are significantly different from each other $(\mathrm{p}<0.001)$. The prostate

Table 2. Clinical characteristics of RARP and LRP groups $(n=170)$

\begin{tabular}{lccc}
\hline \multicolumn{1}{c}{ Variable } & RARP $(\mathrm{n}=93)$ & LRP $(\mathrm{n}=77)$ & $\mathrm{p}$-value \\
\hline Age $(\mathrm{yr})$, median (IQR) & $67(63-71)$ & $68(65-73)$ & 0.149 \\
PSA (ng/mL), median (IQR) & $7.4(5.8-12.2)$ & $8.2(5.5-12.2)$ & 0.175 \\
Prostate volume (mL), median (IQR) & $37.3(27.9-46.3)$ & $35.6(27.9-44.0)$ & 0.197 \\
PSAD (ng/mL $\left.{ }^{2}\right)$, median (IQR) & $0.22(0.16-0.37)$ & $0.24(0.15-0.44)$ & 0.264 \\
Prostate Biopsy & & & \\
Biopsy core number, mean (95\% CI) & $12.6(12.2-13.1)$ & $12.2(11.7-12.7)$ & 0.210 \\
Positive core number, mean (95\% CI) & $5.1(4.4-5.8)$ & $4.2(3.4-4.9)$ & 0.090 \\
Positive rate, mean (95\% CI) & $0.40(0.35-0.46)$ & $0.35(0.29-0.41)$ & 0.228 \\
Residual tumor classification of R1 (\%) & 41.9 & 32.5 & 0.205 \\
\hline
\end{tabular}

RARP: robot-assisted laparoscopic radical prostatectomy, LRP: laparoscopic radical prostatectomy, IQR: interquartile range, PSA: prostate-specific antigen, PSAD: PSA density, CI: confidence interval, R1: microscopic residual tumor. 


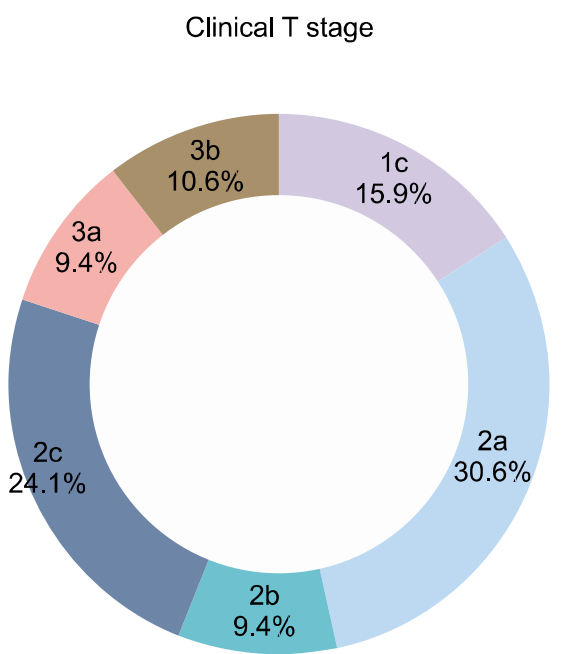

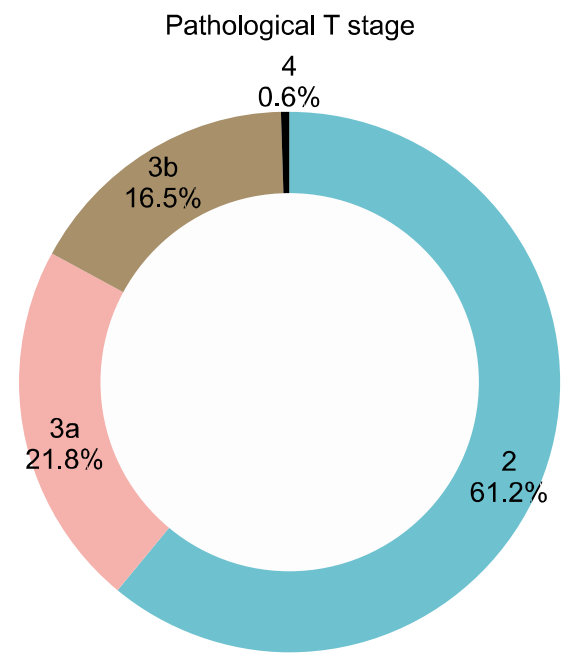

Fig. 1. Distributions of clinical and pathological $\mathrm{T}$ stage of 170 patients.
Table 3. ISUP grade of prostate specimen $(n=170)$

\begin{tabular}{cccc}
\hline ISUP grade & \multicolumn{1}{c}{ Biopsy } & Prostatectomy & p-value \\
\hline 1 & $71(41.8)$ & $28(16.5)$ & $<0.001$ \\
2 & $38(22.4)$ & $85(50.0)$ & \\
3 & $16(9.4)$ & $43(25.3)$ & \\
4 & $33(19.4)$ & $5(2.9)$ & \\
5 & $12(7.1)$ & $9(5.3)$ & \\
\hline
\end{tabular}

Values are presented as number (\%).

ISUP: International Society of Urological Pathology.

Table 4. Risk factors for ECE in $\mathrm{cT} 1-2$ patients $(n=136)$

\begin{tabular}{lrlr}
\hline \multicolumn{1}{c}{ Variable } & Odds ratio & \multicolumn{1}{c}{$95 \%$ CI } & p-value \\
\hline PSA & 1.14 & $0.99-1.32$ & 0.074 \\
PSAD & 0.05 & $0.00-1.11$ & 0.058 \\
Positive biopsy core rate & 32.08 & $4.89-210.28$ & $<0.001$ \\
\hline
\end{tabular}

ECE: extracapsular extension, cT1-2: clinical T stage 1 and 2, CI: confidence interval, PSA: prostate-specific antigen, PSAD: PSA density.

samples of ISUP grades 1, 4, and 5 are more frequently observed in the preoperative biopsy than in confirmative pathology. After prostatectomy, the proportions of the prostate specimen with ISUP grades 2 and 3 have increased; the ratios of ISUP grades $2 / 3$ specimen are $22.4 \% / 9.4 \%$ in biopsy and $50.0 \% / 25.3 \%$ in prostatectomy, respectively.

The distributions of ISUP grades are similar between RARP and LRP groups $(\mathrm{p}=0.991$ and $\mathrm{p}=0.726$ for biopsy and prostatectomy ISUP grade distribution, respectively).

\section{Performance of MRI Scan Predicting ECE}

Four parameters regarding prediction of ECE were investigated in this study; positive predictive value (PPV), negative predictive value (NPV), sensitivity, and specificity. The PPV and NPV to detect ECE in the final pathological specimen are $75.0 \%$ and $73.9 \%$, respectively. The sensitivity of MRI scan for ECE detection is $40.0 \%$. The specificity, however, is $92.7 \%$ which is higher than the value of sensitivity. We can identify the similar trends of the 4 parameters between RARP and LRP.

\section{Risk Factors for pT3a Stage in cT1-2 Patients}

To identify the risk factors for the pathological ECE in cT1-2 cases, we performed the logistic regression analysis including age, PSA, PSAD, number of systemic biopsy cores, number of positive biopsy cores, positive biopsy core rate, ISUP grade of biopsy samples, prostate volume, and clinical $\mathrm{T}$ stage. Positive biopsy core rate is defined as the ratio of the number of positive biopsy cores over the number of systemic biopsy cores. Clinical T1c, T2a, T2b, and T2c stages are included in the test. The results of the logistic regression analysis are demonstrated in Table 4 . The positive biopsy core rate is the only significant risk factor for pathological capsule invasion in $\mathrm{cT} 1-2$ patients $(\mathrm{p}<$ 0.001). We plotted the ROC curve of positive biopsy core rate to estimate the threshold to predict pathological ECE (Fig. 2). The area under the curve (AUC) is 0.691 with the best cutoff value of 0.275 . 


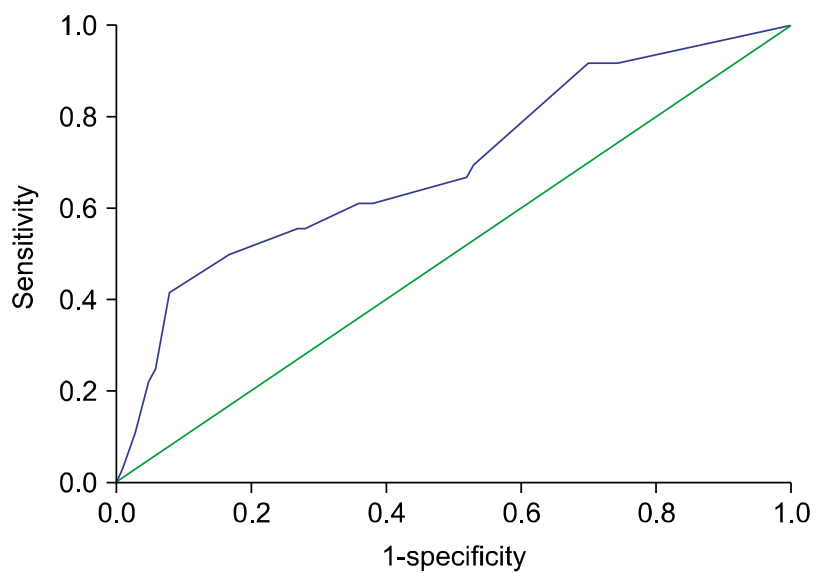

Fig. 2. Receiver-operating characteristic (ROC) curve of positive biopsy core rate in cT1-2 patients. Blue line: positive biopsy core rate, green line: reference line. $\mathrm{p}=0.001$. According to ROC curve (area under the curve $=0.691$ ), the positive biopsy core rate of 0.275 is the cutoff value in this study. The patients with positive biopsy core rate over 0.275 are likely to have a pT3a disease despite of negative image findings for extracapsular extension (sensitivity, 0.611; specificity, 0.620).

\section{DISCUSSION}

The clinical cancer stage is the most important factor for the determination of the best therapeutic option for prostate cancer patients. Particularly, the precise prediction for ECE before prostatectomy is one of the most significant factors. The presence of ECE indicates high-risk disease and the need for aggressive disease management options other than AS.

It is well established that the prevalence of ECE varied from $16.5 \%$ to $53.0 \%$ by the risk groups. ${ }^{18-21}$ In our study, the prevalence of ECE was $21.8 \%$, which is attributed to the high proportion of $\mathrm{cT} 1-2$ patients $(80.0 \%)$ of the cohort. Multiparametric MRI is considered as the best preoperative predictor for ECE at the prostatectomy. ${ }^{20}$ According to Somford et al., ${ }^{20}$ the accuracy of mpMRI for ECE was $73.8 \%$ in overall stages. Sensitivity, specificity, PPV, and NPV were reported as $58.2 \%, 89.1 \%, 84.1 \%$, and $68.3 \%$, respectively. In this study, the accuracy of ECE prediction of mpMRI was $81.2 \%$ with sensitivity, specificity, PPV, and NPV of $40.0 \%, 92.7 \%, 75.0 \%$, and $73.9 \%$, respectively. The accuracy of the MRI tests in our study was higher than in Somford et al.'s due to the lower prevalence $(21.8 \%$ vs. $49.7 \%)$ and high specificity $(92.7 \%$ vs. $89.1 \%)$ of this study. In the analysis of predictive values of mpMRI in the subgroups of different surgical methods, we did not demonstrate any significant differences between RARP and LRP subgroups.

Several clinical factors affect the presence of ECE at prostatectomy: preoperative PSA, biopsy Gleason score, and clinical stages. ${ }^{20,22}$ Studies on ECE in cT1-2 cases confirmed by preoperative MRI scan, however, are limited. In this research, we performed the logistic regression analysis to identify the risk factors for ECE in cT1-2 patients. Age, PSA, PSAD, number of systemic biopsy cores, number of positive biopsy cores, positive biopsy core rate, ISUP grade of biopsy samples, prostate volume, and clinical $\mathrm{T}$ stage were included in the analysis. We demonstrated that positive biopsy core rate was the only significant risk factor for ECE at prostatectomy. PSA, ISUP grade of biopsy samples, and clinical $\mathrm{T}$ stage were not proven to be risk factors in this study. Our cohort of ECE risk factor analysis was limited to the cT1-2 patients, so the risk factors for ECE could be different compared to the literature.

For the identified risk factor, positive biopsy core rate, we plotted ROC curve and demonstrated the positive biopsy core rate of 0.275 as the best threshold value. AUC, sensitivity, and specificity were $0.691,0.611$, and 0.620 , respectively. Thus, patients with positive core rate over 0.275 may have a pathological ECE, although capsular invasion is not expected based on MRI findings. The ratio of ECE at prostatectomy was higher in the positive biopsy core rate over the 0.275 subgroup (36.7\%) than below the 0.275 subgroup $(18.4 \% ; \mathrm{p}=0.017)$.

This study does have limitations. First, we could not build the predictive model for ECE risk factor using the mpMRI parameters. In the logistic regression analysis including PI-RADS score as an independent variable, PI-RADS score was identified as a significant risk factor with p-value of 0.021 . The goodness of fit of this model, however, was poor with $\mathrm{p}<0.001$. The positive biopsy core rate, the only significant risk factor for ECE in this study, was not a predictor directly associated with prostate images. Second, the analysis on the correlation between the locations of pathologic ECE and positive biopsy cores could not be conducted due to the absence of locational information of ECE on the confirmative pathologic reports. Finally, this was a single center retrospective study with the unavoidable limitations of such kinds. 


\section{CONCLUSIONS}

Multiparametric MRI can be a reasonable diagnostic tool to predict ECE in the preoperative period. The patients with high positive core rate are associated with ECE at prostatectomy though the prostatic capsular invasion is not proven in MRI scan.

\section{CONFLICT OF INTEREST}

The authors claim no conflicts of interest.

\section{REFERENCES}

1. Bray F, Ferlay J, Soerjomataram I, Siegel RL, Torre LA, Jemal A. Global cancer statistics 2018: GLOBOCAN estimates of incidence and mortality worldwide for 36 cancers in 185 countries. CA Cancer J Clin 2018;68:394-424.

2. Wilt TJ, Brawer MK, Jones KM, Barry MJ, Aronson WJ, Fox S, et al. Radical prostatectomy versus observation for localized prostate cancer. N Engl J Med 2012;367:203-13.

3. Bill-Axelson A, Holmberg L, Garmo H, Rider JR, Taari $\mathrm{K}$, Busch C, et al. Radical prostatectomy or watchful waiting in early prostate cancer. N Engl J Med 2014;370:93242.

4. Tosoian JJ, Carter HB, Lepor A, Loeb S. Active surveillance for prostate cancer: current evidence and contemporary state of practice. Nat Rev Urol 2016;13:205-15.

5. Niu XK, Chen ZF, Chen L, Li J, Peng T, Li X. Clinical application of biparametric MRI texture analysis for detection and evaluation of high-grade prostate cancer in zone-specific regions. AJR Am J Roentgenol 2018;210: 549-56.

6. Bratan F, Niaf E, Melodelima C, Chesnais AL, Souchon $\mathrm{R}$, Mège-Lechevallier $\mathrm{F}$, et al. Influence of imaging and histological factors on prostate cancer detection and localisation on multiparametric MRI: a prospective study. Eur Radiol 2013;23:2019-29.

7. Le JD, Tan N, Shkolyar E, Lu DY, Kwan L, Marks LS, et al. Multifocality and prostate cancer detection by multiparametric magnetic resonance imaging: correlation with whole-mount histopathology. Eur Urol 2015;67:569-76.

8. Frye TP, George AK, Kilchevsky A, Maruf M, Siddiqui $\mathrm{MM}$, Kongnyuy $\mathrm{M}$, et al. Magnetic resonance imagingtransrectal ultrasound guided fusion biopsy to detect progression in patients with existing lesions on active surveillance for low and intermediate risk prostate cancer. J Urol 2017;197:640-6.
9. Bryant RJ, Yang B, Philippou Y, Lam K, Obiakor M, Ayers $J$, et al. Does the introduction of prostate multiparametric magnetic resonance imaging into the active surveillance protocol for localized prostate cancer improve patient re-classification? BJU Int 2018;122:794-800.

10. Da Rosa MR, Milot L, Sugar L, Vesprini D, Chung H, Loblaw A, et al. A prospective comparison of MRI-US fused targeted biopsy versus systematic ultrasound-guided biopsy for detecting clinically significant prostate cancer in patients on active surveillance. J Magn Reson Imaging 2015;41:220-5.

11. Stamatakis L, Siddiqui MM, Nix JW, Logan J, RaisBahrami S, Walton-Diaz A, et al. Accuracy of multiparametric magnetic resonance imaging in confirming eligibility for active surveillance for men with prostate cancer Cancer 2013;119:3359-66.

12. Weinreb JC, Barentsz JO, Choyke PL, Cornud F, Haider MA, Macura KJ, et al. PI-RADS Prostate Imaging Reporting and Data System: 2015, Version 2. Eur Urol 2016;69:16-40.

13. Park SY, Jung DC, Oh YT, Cho NH, Choi YD, Rha KH, et al. Prostate Cancer: PI-RADS version 2 helps preoperatively predict clinically significant cancers. Radiology 2016;280:108-16.

14. Barentsz JO, Weinreb JC, Verma S, Thoeny HC, Tempany $\mathrm{CM}$, Shtern F, et al. Synopsis of the PI-RADS v2 guidelines for multiparametric prostate magnetic resonance imaging and recommendations for use. Eur Urol 2016;69: 41-9.

15. Purysko AS, Bittencourt LK, Bullen JA, Mostardeiro TR, Herts BR, Klein EA. Accuracy and interobserver agreement for Prostate Imaging Reporting and Data System, Version 2, for the characterization of lesions identified on multiparametric MRI of the prostate. AJR Am J Roentgenol 2017;209:339-49.

16. Bezinque A, Moriarity A, Farrell C, Peabody H, Noyes SL, Lane BR. Determination of prostate volume: a comparison of contemporary methods. Acad Radiol 2018;25: 1582-7.

17. Edge SB, Byrd DR, Compton CC, Fritz AG, Greene FL, Trotti A, editors. AJCC cancer staging manual. 7th ed. New York: Springer; 2010. p. 457-68.

18. Tewari AK, Srivastava A, Sooriakumaran P, Grover S, Desir S, Dev H, et al. Pathological outcomes and strategies to achieve optimal cancer control during robotic radical prostatectomy in Asian-Indian men. Indian J Urol 2011;27: 326-30.

19. Yossepowitch O, Eggener SE, Bianco FJ Jr, Carver BS, Serio A, Scardino PT, et al. Radical prostatectomy for clinically localized, high risk prostate cancer: critical analysis of risk assessment methods. J Urol 2007;178:493-9. 20. Somford DM, Hamoen EH, Fütterer JJ, van Basten JP, 
Hulsbergen-van de Kaa CA, Vreuls W, et al. The predictive value of endorectal 3 Tesla multiparametric magnetic resonance imaging for extraprostatic extension in patients with low, intermediate and high risk prostate cancer. J Urol 2013;190:1728-34.

21. Nakanishi H, Troncoso P, Babaian RJ. Prediction of extraprostatic extension in men with biopsy Gleason score of
8 or greater. J Urol 2008;180:2441-5.

22. Joniau S, Hsu CY, Lerut E, Van Baelen A, Haustermans $\mathrm{K}$, Roskams $\mathrm{T}$, et al. A pretreatment table for the prediction of final histopathology after radical prostatectomy in clinical unilateral T3a prostate cancer. Eur Urol 2007; 51:388-94. 